\title{
Synthetic Loading for Symmetrical and Asymmetrical Nine-phase Machines
}

\author{
A. A. Abduallah, O. Dordevic, M. Jones, E. Levi \\ Department of Electronics and Electrical Engineering, \\ Liverpool John Moores University, \\ Liverpool, UK
}

\begin{abstract}
Substantial amount of research and development have been conducted in relation to multiple three-phase winding machines. This is due to their reliability and fault tolerant capabilities. Development of these machines requires a few tests in order to validate machine's design and characteristics. The fullload test is one of the most important tests. During this test the machine's efficiency and thermal design can be evaluated under various conditions. In this paper, a new approach of conducting the full-load test of nine-phase machine is presented. The main advantage of the proposed scheme is that there is no need for mechanical coupling of the tested machine with another one. Usually, the machine is coupled with another machine, of the same or higher power rating, which is used as a load. The proposed test utilises indirect rotor field-oriented control (IRFOC) and vector space decomposition (VSD) to achieve the desired loading and hence temperature-rise in the machine. The ability to recirculate the power among the winding sets of the nine-phase machine is an advantage of the proposed scheme. In other words, the machine is loaded using its own winding sets - hence the test is classified as synthetic loading. The proposed scheme is validated through simulation results.
\end{abstract}

Keywords - Synthetic loading; Nine-phase machine; Multiple three-phase machine; IRFOC.

\section{INTRODUCTION}

High power applications often utilise multiphase machines (more than three phases), especially those with multiple threephase winding sets [1-3]. Nowadays they are becoming more and more used, especially in the renewable energy sector. This is because of their fault-tolerant capabilities and their wellestablished three-phase power electronics technology [4-7]. The main advantage of using multiphase machines is their ability to split up the power among the $n$ phases $(n>3)$. Other advantages, which mostly come from an increased number of degrees of freedom present in multiphase machines, are stated in [1-3].

Multiple three-phase winding machines can be modelled using two different approaches: multi-stator (MS) and vector space decomposition (VSD) approach. MS approach considers each three-phase winding set as a separate three-phase machine [8]. The main benefit of this modelling approach is the ability to control each three-phase winding set separately. By using appropriately aligned Clarke's transformation for each threephase winding, each set has its own flux and torque $d q$ current components. However, the problem with this approach is that the winding set equations are not completely decoupled. On the other hand, VSD modelling approach observes the machine as a whole, taking into account coupling between all phases. It provides a clear mapping of the harmonics and only a single flux/torque producing subspace is present. The drawback of this approach is the loss of the ability to control each winding set separately. IRFOC can be implemented using both approaches for multiple three-phase winding machines.

Recently, the pace of design and development of new multiphase machines have increased. To validate the machine's design, the full-load test (temperature-rise test) should be conducted. This test is important to verify the machine's performance and thermal design before commissioning it. Usually, the machine's thermal design is tested using finite element method (FEM) software, at first. However, huge computational time and powerful computers are needed. Furthermore, the machine model must be very precise to obtain accurate results. To avoid any inaccuracy in the machine model, the practical and experimental tests are preferable.

The back-to-back configuration is one of the most common full-load test configurations used to experimentally test the machine thermal design $[9,10]$. The main advantage of this test is the ability to recirculate the power if the dc-links of the power electronic converters are connected. However, the test requires another machine with the same or higher power rating than the tested machine. The shafts of these machines should be mechanically coupled to each other. Overall, this requires huge resources and time. Therefore, alternative methods to achieve the temperature-rise test are required.

Several authors have introduced different methods and techniques to achieve the temperature-rise test without the need to mechanically couple two machines [11-17]. Some of the suggested methods are two-frequency method [14], phantom loading [11], and inverter driven methods [12]. Although the machine temperature-rise goal can be achieved using the previous methods, the power supplied to the machine is not retrievable. This is the main drawback of the previous methods. This will cost substantial amount of energy for high power machines. Thus, a new approach where the ability to recirculate the power, without the need for another machine, is introduced.

A new approach for testing the machine's full-load capabilities is introduced in $[18,19]$. The approach is capable of loading the machine and re-circulating the power simultaneously. It uses sensorless four-quadrant field-oriented control. The machine in $[18,19]$ is of specific construction with segmented stator. Four three-phase segments exist and opposite 
segments are connected together to one of the two converters. Depending on the mode of operation (motoring or generation), the control variable of connected segments is changed (speed or torque). The dc-links are connected, so that the power is circulated between the segments.

In this paper, a new approach for implementing the full-load test for symmetrical and asymmetrical nine-phase machines is introduced. The approach utilises the IRFOC scheme and VSD modelling approach to achieve the temperature rise. The scheme injects fundamental frequency currents into machine's $x-y$ circuits to control the winding set currents individually. The approach varies the winding set operating conditions between no-load condition, motoring and generation, while maintaining the reference speed.

The paper consists of the following sections. Section II presents the correlations between the MS and VSD modelling approaches for symmetrical and asymmetrical nine-phase machine. Next, section III introduces synthetic loading technique for nine-phase machines using multiple vector control. In section IV, the synthetic loading utilising the VSD and IRFOC is introduced. The simulation results are presented in section V. Finally, the conclusions are given in section VI.

\section{MS AND VSD CORRELATIONS}

The synthetic loading approach presented in [18, 19] for segmented stator machines is based on controlling each segment separately with different control variables. Two segments run in speed mode (motoring) and the remaining two are in the torque mode (generation). This principle of synthetic loading can be applied to multiphase machines with multiple three-phase winding sets using MS approach.

The synthetic loading using multiple vector control and MS modelling approach can be applied for nine-phase machines in the same manner as in [18, 19]. However, controlling multiphase machines based on MS modelling approach is not recommended since there is coupling between the winding sets. The equivalent circuit representation of nine-phase induction machines, in triple $d-q$ reference frame, is illustrated in Fig. 1. Controlling multiphase machines is recommended using the

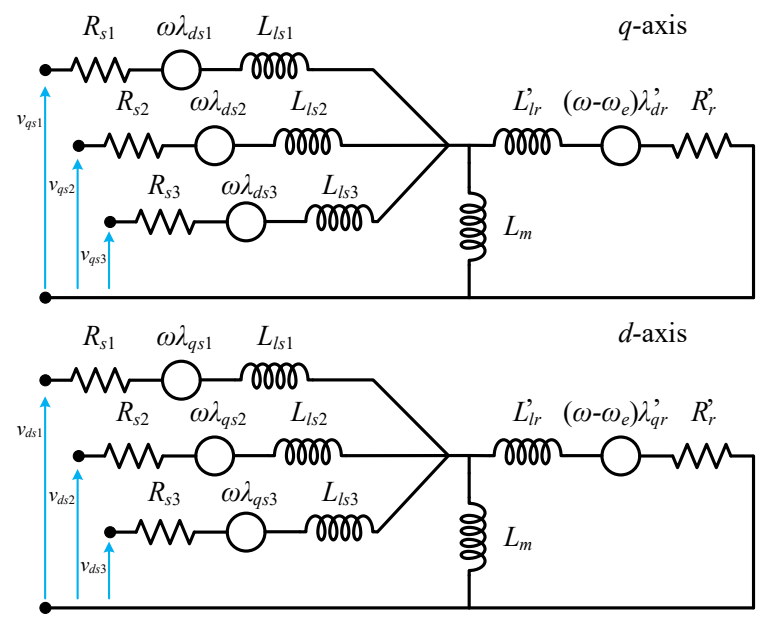

Fig. 1: Equivalent circuit representation of nine-phase machines using MS approach.
VSD approach. This approach decouples the machine into $n / 2$ subspaces. Implementation of synthetic loading for the ninephase machine, by using the VSD approach only, is not possible because the VSD approach does not hold the information about the individual winding sets. Therefore, a combination of both approaches is necessary. Additionally, the cross coupling between the winding sets is eliminated.

A combination of both modelling approaches is presented in [20], where the correlations between MS and VSD approach are established. In other words, the links between the individual winding set currents $\alpha_{i}-\beta_{i}$ and VSD $x-y$ currents are found. The multiple vector control using MS approach for a nine-phase machine can be implemented utilising Clarke's transformation matrix for three-phase machines. However, the displacement of the winding sets with respect to the first winding set should be taken into consideration, by means of:

$$
[\mathrm{C}(\delta)]=\frac{2}{3}\left[\begin{array}{c:ccc}
\alpha_{i} & \cos (\delta) & \cos (\delta+2 \pi / 3) & \cos (\delta+4 \pi / 3) \\
\beta_{i} & \sin (\delta) & \sin (\delta+2 \pi / 3) & \sin (\delta+4 \pi / 3) \\
o_{i} & \frac{1}{2} & \frac{1}{2} & \frac{1}{2}
\end{array}\right]
$$

where $\delta$ represents the spatial displacement between the winding sets. The multiple vector control can be applied to both symmetrical and asymmetrical nine-phase machines utilising (1). For a symmetrical nine-phase machine, $\delta=0$ for the first winding set. For the second and third winding set, $\delta=2 \pi / 9$ and $\delta=4 \pi / 9$, respectively. The spatial displacements for an asymmetrical nine-phase machine are $\delta=0, \delta=\pi / 9$ and $\delta=2 \pi / 9$, for the first, second and the third winding set, respectively. Fig. 2 illustrates the magnetic axes of the nine-phase machines in symmetrical and asymmetrical configuration.

As mentioned earlier, VSD modelling approach provides complete decoupling among $n / 2$ subspaces. For high performance control of multiphase machines, VSD is the preferred choice. The first subspace $(\alpha-\beta)$ controls the flux and the torque produced in the nine-phase machine. The $x-y$ subspaces are loss-producing subspaces (Fig. 3). Usually, in practice, components in these subspaces are controlled to zero to remove any asymmetries present in the machine or in the power converter. Sometimes, the $x-y$ currents are controlled to achieve a post-fault operation of the nine-phase machines. The other components $\left(0_{1}, 0_{2}\right.$ and $\left.0_{3}\right)$ are the zero-sequences for each winding set, if the machine has isolated neutral points. The VSD
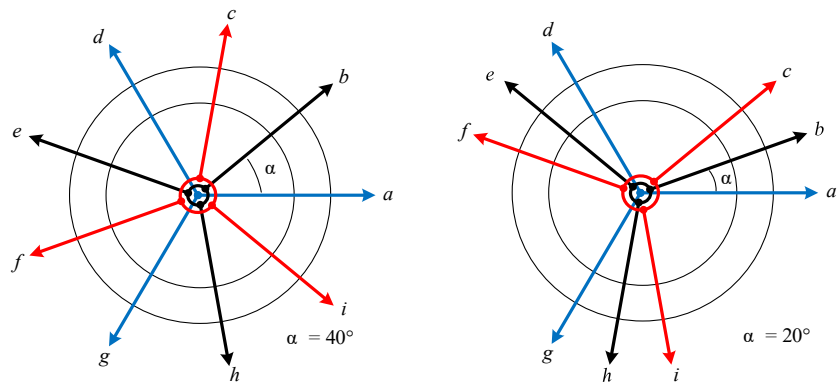

Fig. 2: Magnetic axes of the symmetrical (left) and asymmetrical (right) ninephase machine. 


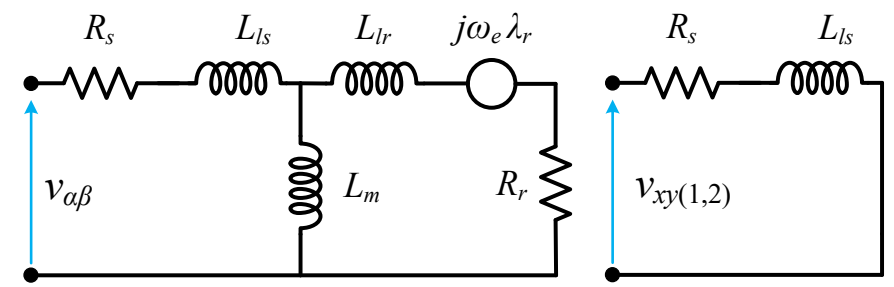

Fig. 3: Equivalent circuit representation of nine-phase induction machines using VSD approach.

transformation matrix for asymmetrical nine-phase machine with three isolated neutral points is defined as:

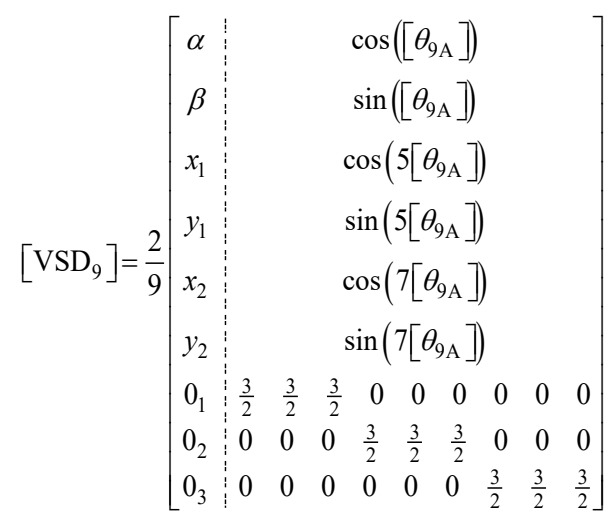

where $\left[\theta_{9 \mathrm{~A}}\right]$ is given with:

$$
\left[\theta_{9 \mathrm{~A}}\right]=\pi / 9 \cdot\left[\begin{array}{lllllllll}
0 & 6 & 12 & 1 & 7 & 13 & 2 & 8 & 14
\end{array}\right]
$$

For a symmetrical nine-phase machine, with three-isolated neutral points, VSD transformation matrix is defined as:

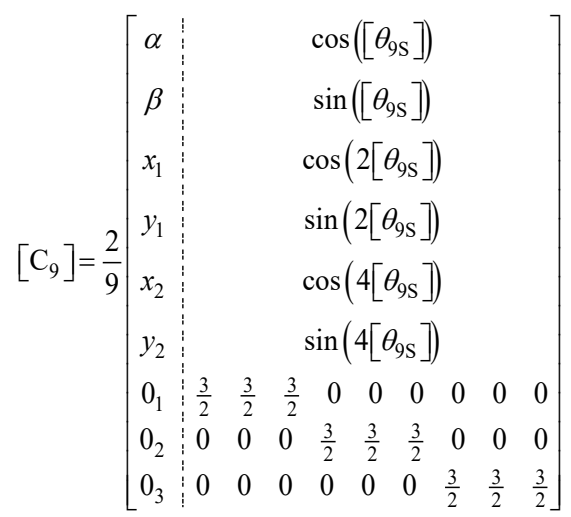

where $\left[\theta_{9 \mathrm{~S}}\right]$ for symmetrical nine-phase machines is:

$$
\left[\theta_{9 \mathrm{~S}}\right]=2 \pi / 9 \cdot\left[\begin{array}{lllllllll}
0 & 3 & 6 & 1 & 4 & 7 & 2 & 5 & 8
\end{array}\right]
$$

Unlike (1), (2)-(5) show that the machine has a single $\alpha-\beta$ subspace. Therefore, the control of the winding sets individually is not possible using these transformations.

The authors of [20] have introduced a current sharing strategy using VSD transformation. The strategy utilises the correlations between MS and VSD approach. The individual winding set currents $\alpha_{i}-\beta_{i}-o_{i}$ are mapped into $x-y$ subspace currents of the VSD representation. This allows individual current control of the winding sets directly from the VSD approach. These correlations can be found for asymmetrical nine-phase machine in the following way. Initially, the relationships between the phase currents and $\alpha_{i}-\beta_{i}-O_{i}$ for the three winding sets should be obtained based on (1):

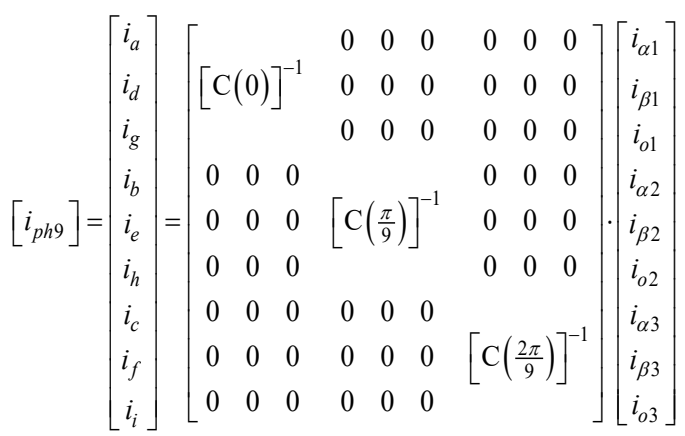

The product in (6) shows the contribution of the three winding sets to the phase currents. By multiplying $\left[i_{p h}\right]$ from (6) by [VSD 9 ] from (2), the product will define how the VSD currents are related to the individual winding set currents $\left(\alpha_{i}-\beta_{i}-O_{i}\right)$. One can find that the final links between MS and VSD approach are:

$$
\left[i_{\mathrm{VSD} 9}\right]=\left[\begin{array}{c}
i_{\alpha} \\
i_{\beta} \\
i_{x 1} \\
i_{y 1} \\
i_{x 2} \\
i_{y 2} \\
i_{01} \\
i_{02} \\
i_{03}
\end{array}\right]=\left[\mathrm{VSD}_{9}\right] \cdot\left[i_{p h 9}\right]=\left[\begin{array}{c}
\frac{1}{3}\left(i_{\alpha 1}+i_{\alpha 2}+i_{\alpha 3}\right) \\
\frac{1}{3}\left(i_{\beta 1}+i_{\beta 2}+i_{\beta 3}\right) \\
\frac{1}{6}\left(2 i_{\alpha 1}-i_{\alpha 2}-i_{\alpha 3}+\sqrt{3}\left(i_{\beta 2}-i_{\beta 3}\right)\right) \\
\frac{1}{6}\left(\sqrt{3}\left(i_{\alpha 2}-i_{\alpha 3}\right)-i_{\beta 1}+i_{\beta 2}+i_{\beta 3}\right) \\
\frac{1}{6}\left(2 i_{\alpha 1}-i_{\alpha 2}-i_{\alpha 3}-\sqrt{3}\left(i_{\beta 2}-i_{\beta 3}\right)\right) \\
\frac{1}{6}\left(\sqrt{3}\left(i_{\alpha 2}-i_{\alpha 3}\right)+i_{\beta 1}-i_{\beta 2}-i_{\beta 3}\right) \\
i_{o 1} \\
i_{o 2} \\
i_{o 3}
\end{array}\right]
$$

Note that the result in (7) is the same for both asymmetrical and symmetrical nine-phase machine with three isolated neutral points. To get (7) into a compact form, the following complex notation is used:

$$
\begin{aligned}
& \bar{i}_{\alpha \beta}=i_{\alpha}+j i_{\beta}=I_{\alpha \beta} e^{j \varphi_{\alpha \beta}} \\
& \bar{i}_{x y(1,2)}=i_{x(1,2)}+j i_{y(1,2)}=I_{x y(1,2)} e^{j \varphi_{x y(1,2)}} \\
& \bar{i}_{\alpha \beta(1,2,3)}=i_{\alpha(1,2,3)}+j i_{\beta(1,2,3)}=I_{\alpha \beta(1,2,3)} e^{j \varphi_{\alpha \beta(1,2,3)}}
\end{aligned}
$$

Based on (8), the current space vectors can be expressed in terms of the winding set current space vectors. Consequently, (7) becomes:

$$
\begin{aligned}
& \bar{i}_{\alpha \beta}=\frac{1}{3}\left(I_{\alpha \beta 1} e^{j \varphi_{\alpha \beta 1}}+I_{\alpha \beta 2} e^{j \varphi_{\alpha \beta 2}}+I_{\alpha \beta 3} e^{j \varphi_{\alpha \beta 3}}\right) \\
& \bar{i}_{x y 1}=\frac{1}{3}\left(I_{\alpha \beta 1} e^{-j \varphi_{\alpha \beta 1}}+I_{\alpha \beta 2} e^{-j \varphi_{\alpha \beta 2}} e^{j 2 \pi / 3}+I_{\alpha \beta 3} e^{-j \varphi_{\alpha \beta 3}} e^{-j 2 \pi / 3}\right) \\
& \bar{i}_{x y 2}=\frac{1}{3}\left(I_{\alpha \beta 1} e^{j \varphi_{\alpha \beta 1}}+I_{\alpha \beta 2} e^{j \varphi_{\alpha \beta 2}} e^{j 2 \pi / 3}+I_{\alpha \beta 3} e^{j \varphi_{\alpha \beta 3}} e^{-j 2 \pi / 3}\right)
\end{aligned}
$$

By introducing the current sharing coefficients $K_{1}, K_{2}$ and $K_{3}$, the winding set current space vectors can be rewritten in terms of $\alpha-\beta$ space vector. The following relationships are defined:

$$
\bar{i}_{\alpha \beta 1}=K_{1} \bar{i}_{\alpha \beta} \quad \bar{i}_{\alpha \beta 2}=K_{2} \bar{i}_{\alpha \beta} \quad \bar{i}_{\alpha \beta 3}=K_{3} \bar{i}_{\alpha \beta}
$$

By substituting newly introduced current sharing coefficients of (10) into (9), the following relationships between $\alpha-\beta$ space vectors and winding set current space vectors can be obtained: 


$$
\begin{aligned}
& \bar{i}_{\alpha \beta}=\frac{1}{3}\left(K_{1}+K_{2}+K_{3}\right) \bar{i}_{\alpha \beta} \\
& \bar{i}_{x y 1}=\frac{1}{3}\left(K_{1}+K_{2} e^{j 2 \pi / 3}+K_{3} e^{-j 2 \pi / 3}\right) \bar{i}_{\alpha \beta}^{*} \\
& \bar{i}_{x y 2}=\frac{1}{3}\left(K_{1}+K_{2} e^{j 2 \pi / 3}+K_{3} e^{-j 2 \pi / 3}\right) \bar{i}_{\alpha \beta}
\end{aligned}
$$

The control of the current of each winding set is now possible by using the relationships introduced in (11). However, this sharing method depends on the total electromechanical energy conversion, defined by $i_{\alpha \beta}$. Thus, in order to use this strategy, a load on the shaft should be applied to test the machine's thermal design. Alternative methods for implementing the temperaturerise test are introduced in the following sections.

\section{Synthetic LOAding OF Nine-Phase MACHINES Using MULTIPLE VECTOR CONTROL}

The temperature-rise test can be applied for nine-phase machines with three neutral points using the same strategy as in [18], where each winding set is considered as a segment. Multiple vector control can be applied to each winding set with a different control variable. The control variable is dependent on the mode of operation of the winding set (motoring or generation). The motoring winding set would have the speed, while the winding sets in generation would have the torque as the control variable. Since there is an odd number of the winding sets in the nine-phase machines, it is not possible to put one half of the sets in motoring and another half in generation mode as in $[18,19]$. On the other hand, if two sets are in say motoring and one in generation mode, then different currents would be flowing in the winding sets. Therefore, alternation of the operation mode for the winding sets should be considered.

The temperature-rise test for nine-phase machines utilising multiple vector control can be implemented as follows:

1. During the initial acceleration of the machine, the three winding sets are set to motoring mode (speed-controlled).

2. After reaching steady-state, one of the winding sets is set to generation mode (torque-controlled) by applying a negative synthetic load torque reference $\left(T_{l}^{*}\right)$.

3. Since there is an odd number of winding sets, alteration of the torque-controlled winding set, among all of them, is applied.

The current amplitude can be controlled in the motoring winding sets by changing the coefficients $G_{1}, G_{2}$ and $G_{3}$ which

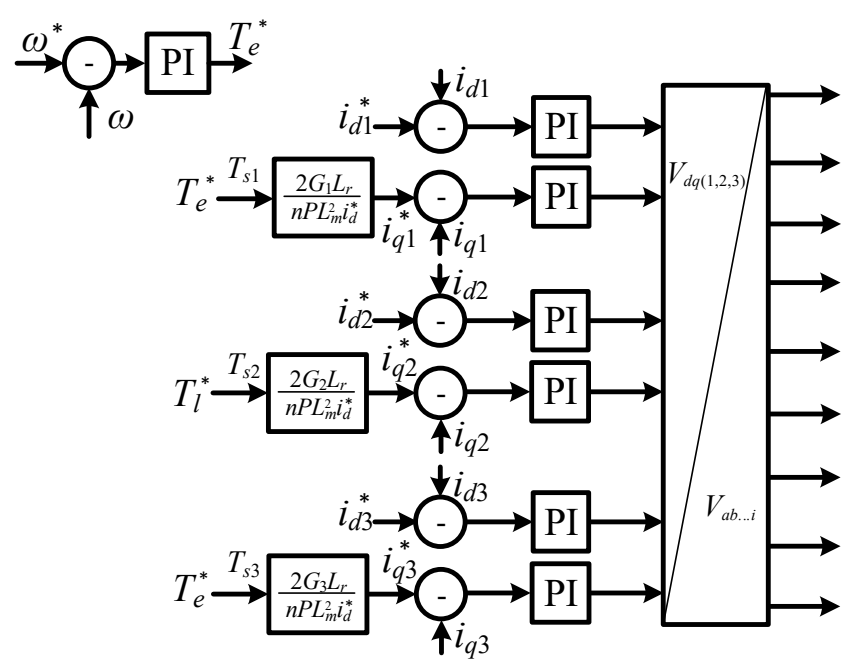

Fig. 4: Synthetic loading schematic using multiple vector control for nine-phase induction machines.

are illustrated in the control schematic shown in Fig. 4. In other words, the developed torque by each winding set can be controlled by changing these coefficients. The equilibrium torque is expressed by the following equation:

$$
2 T_{s 1}=-T_{s 2}=2 T_{s 3}=T_{a g}
$$

where $T_{s 1}, T_{s 2}$ and $T_{s 3}$ stand for the developed torque by the first, second and the third winding set, respectively. $T_{a g}$ stands for the developed torque in the air gap. As mentioned earlier, the crosscoupling between the winding sets is the main drawback of multiple vector control. Therefore, the synthetic loading using VSD is presented next.

\section{SyNTHETIC LOADING OF NINE-PHASE MACHINES USING IRFOC AND VSD}

The implementation of the synthetic loading test using VSD is possible utilising the auxiliary currents and the current sharing strategy shown in section II. The synthetic loading schematic for a nine-phase induction machine is illustrated in Fig. 5. The configuration is equally applicable to symmetrical and asymmetrical nine-phase machines.

The aim of the test is to achieve the desired temperature rise of the machine. The temperature change is caused by the losses

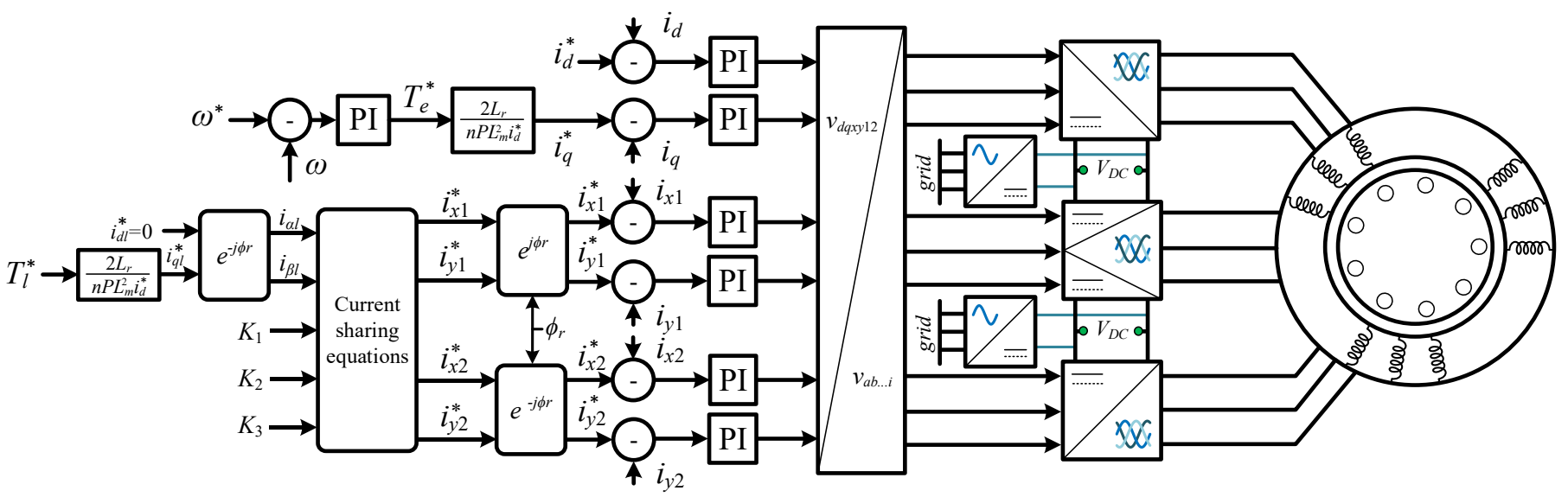

Fig. 5: Synthetic loading schematic using IRFOC and VSD for (asymmetrical) nine-phase induction machine. 
in the machine. Therefore, the machine should be run under the rated conditions in order to properly conduct the test. As discussed, the nine-phase machine has an odd number of winding sets. Therefore, the number of motoring winding sets cannot be equal to the number of generation winding sets. One has two options, either to divide the motoring power between two winding sets, or to use one winding set for motoring and another for generation while the third winding set is in no-load mode. The second option is chosen here. Therefore, the synthetic loading test can be applied using the following steps:

1. During the initial acceleration of the nine-phase machine, the auxiliary currents control is off.

2. After the machine reached the reference speed, the synthetic loading test is started by applying $T_{l}^{*}$ and the current sharing coefficients $K_{i}$.

3. The motoring, generation and no-load winding sets are exchanged periodically during the test by changing $K_{i}$.

The current sharing coefficients $K_{i}$ are able to change the current's amplitude and direction. This happens when the coefficients are defined based on the electromechanical subspace's current $\alpha-\beta$. However, in the implementation of the proposed synthetic loading test, the coefficients are defined based on $i_{\alpha \beta l}$ (see Fig. 5). Thus, the current sharing equations (11) can be rewritten as:

$$
\begin{aligned}
& \bar{i}_{x y 1}=\frac{1}{3}\left(K_{1}+K_{2} e^{j 2 \pi / 3}+K_{3} e^{-j 2 \pi / 3}\right) \bar{i}_{\alpha \beta l}^{*} \\
& \bar{i}_{x y 2}=\frac{1}{3}\left(K_{1}+K_{2} e^{j 2 \pi / 3}+K_{3} e^{-j 2 \pi / 3}\right) \bar{i}_{\alpha \beta l}
\end{aligned}
$$

By applying $T_{l}^{*}$ and the appropriate sharing coefficients $K_{i}$, the temperature-rise of the machine can be obtained.

\section{Simulation Results}

The proposed synthetic loading approach using IRFOC and VSD for nine-phase machines is validated through simulations in Matlab/Simulink. The control schematic illustrated in Fig. 5 is implemented for symmetrical nine-phase induction machine. The nine-phase machine parameters are shown in Table I. Simulation results of the synthetic loading for a nine-phase induction machine are illustrated in Fig. 6. Initially, the machine is magnetised for $1.5 \mathrm{sec}$. Afterwards, the machine speed reference is set to $250(\mathrm{rad} / \mathrm{sec})$. When the machine has reached steady state, the synthetic loading test is started (at $t=4 \mathrm{sec}$ ) by changing $T_{l}^{*}$ from zero to $10 \mathrm{Nm}$. Synthetic loading is applied by imposing the auxiliary currents according to (13). The sharing coefficients are defined as follows: $K_{i}=1$ represents motoring winding set, $K_{i}=-1$ represents generation winding set and $K_{i}=0$ represents no-load winding set. After applying $T_{l}{ }^{*}$, the sharing coefficients $K_{i}$ are altered between 1, 0 and -1. Each set is changing between motoring and no-load mode of operation (with trapezoidal change of $K_{i}$ ), staying in each mode for $0.1 \mathrm{sec}$. After repeating this ten times, i.e. after $2 \mathrm{sec}$ in total, the set goes to generation mode for another $1 \mathrm{sec}$. The sequence repeats in the way illustrated in the current waveforms in Fig. 6. Note that when the set is in no-load mode, $K_{i}=0$, the current of the set is not zero but rather it is equal to the no-load current. This is because the coefficients $K_{i}$ are sharing the synthetic torque and $\alpha \beta_{l}$ currents, rather than the real electromechanical torque and real $\alpha \beta$ currents.
Table I: Nine-phase induction machine parameters.

\begin{tabular}{|l|c|l|c|}
\hline$f_{\text {switching }}$ & $10 \mathrm{kHz}$ & $2 P$ & 2 (one pair) \\
\hline$L_{l r}$ & $11 \mathrm{mH}$ & $R_{r}$ & $2 \Omega$ \\
\hline$L_{l s}$ & $24 \mathrm{mH}$ & $R_{s}$ & $5.3 \Omega$ \\
\hline$L_{m}$ & $520 \mathrm{mH}$ & $V_{D C}$ & $750 \mathrm{~V}$ \\
\hline
\end{tabular}
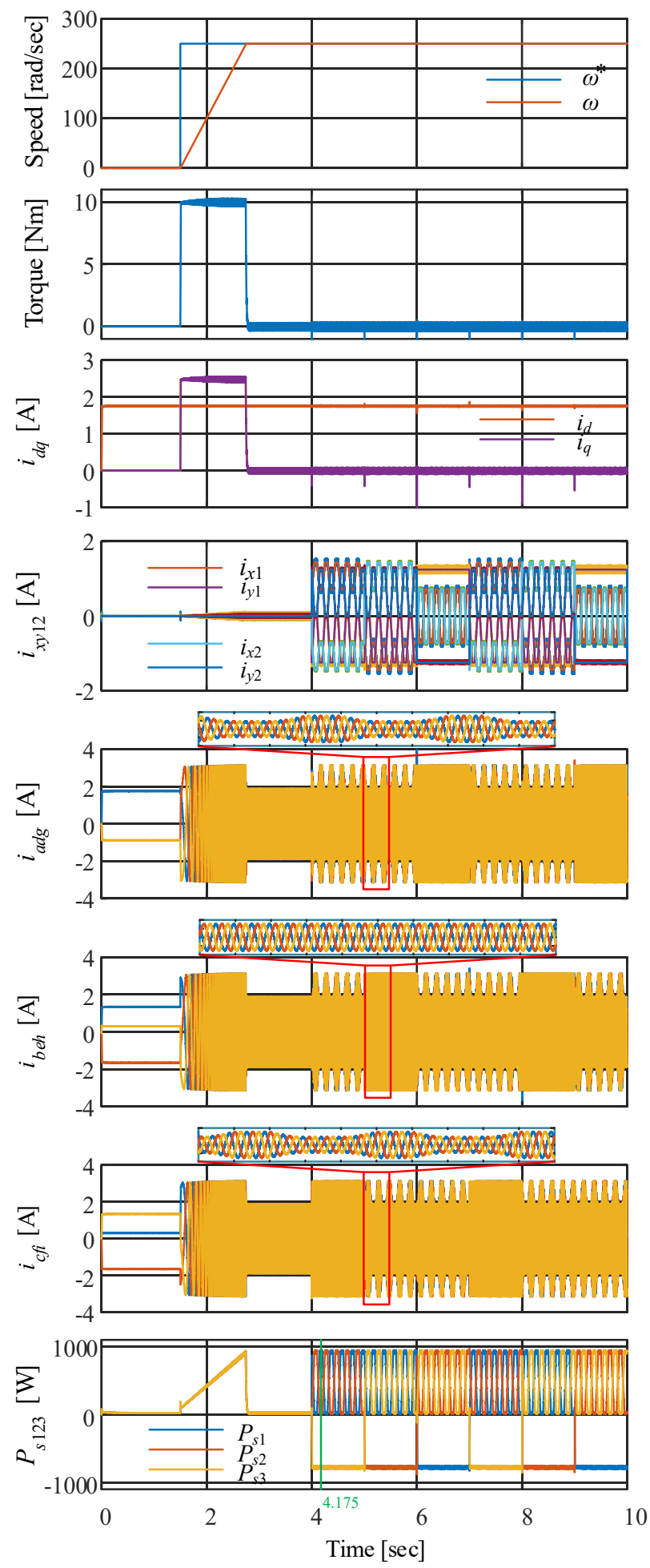

Fig. 6: Synthetic loading simulation results for symmetrical nine-phase induction machines. 
When sets are operating with $K_{i}=1$ and $K_{i}=-1$, the currents obtained correspond to the rated currents. From Fig. 6, one can note that the torque on the shaft, during the synthetic loading process, remains stable at zero. The flux and the torque producing currents $\left(i_{d}\right.$ and $\left.i_{q}\right)$ are constant during the test. Note that, because there is no physical load on the shaft, $i_{q}=0$. The winding set input powers $\left(P_{s 123}\right)$ shown in Fig. 6 (bottom subplot) illustrate the ability of the test to re-circulate the power among the winding sets and to emulate the nominal conditions of the machine. The graph with the input power of the winding sets, shows that the difference between the motoring and the generation winding set power is equivalent to the total copper losses of both winding sets. For example, looking at instant at $t=4.175 \mathrm{sec}$, the power consumed by the first set is $P_{s 1}=$ 923.24 W (motoring mode) and by the second set $P_{s 2}=25.45 \mathrm{~W}$ (no-load, hence consumed for the copper losses only). The third set is in generation mode with $P_{s 3}=-777.319 \mathrm{~W}$. Therefore, the total power losses are $171.37 \mathrm{~W}$. The total copper losses can be calculated from the winding sets in motoring and generation mode $\left(6 \cdot R_{s} \cdot 2.1517^{2}=147.27 \mathrm{~W}\right)$ plus the winding set in no-load mode $\left(3 \cdot R_{s} \cdot 1.241^{2}=24.48 \mathrm{~W}\right)$ which is equal to $171.75 \mathrm{~W}$. The windage and friction losses as well as power electronic converter losses are neglected in the simulation.

\section{CONCLUSION}

In this paper, synthetic loading test for nine-phase machines has been presented. The test utilises VSD and IRFOC and can be used to validate the efficiency and temperature-rise of the nine-phase machines. The test is based on the current sharing strategy among the three-phase winding sets of the machine. The power is circulated between the winding sets. The currents of each winding set are shared to achieve the values corresponding to the nominal conditions of the machine. The winding sets alternate between three modes of operation: motoring, generation and no-load. During the motoring and generation mode, the rated current flows through the phases and the losses are at their maximum. The power is recirculated among the winding sets by connecting the dc-links of the converters. The power taken from the grid is equal to the overall losses in the system. The requirement for mechanical coupling and another machine to test the rated conditions of the ninephase machine does not exist anymore when using this approach. The simulation results validate the ability of the approach to test the performance and thermal design of the machine.

\section{ACKNOWLEDGMENT}

The authors would like to acknowledge the Engineering and Physical Sciences Research Council (EPSRC) for supporting the "Power flow control in future electric vehicles and dc microgrids with multiple energy sources" project (EP/P00914X/1).

\section{REFERENCES}

[1] E. Levi, R. Bojoi, F. Profumo, H. A. Toliyat, and S. Williamson, "Multiphase induction motor drives - a technology status review," IET Electric Power Applications, vol. 1, no. 4, pp. 489-516, 2007.
[2] E. Levi, "Multiphase electric machines for variable-speed applications," IEEE Transactions on Industrial Electronics, vol. 55, no. 5, pp. 18931909, 2008.

[3] E. Levi, "Advances in converter control and innovative exploitation of additional degrees of freedom for multiphase machines," IEEE Transactions on Industrial Electronics, vol. 63, no. 1, pp. 433-448, 2016.

[4] H. S. Che, W. P. Hew, N. A. Rahim, E. Levi, M. Jones, and M. J. Duran, "Current control of a six-phase induction generator for wind energy plants," in $12^{\text {th }}$ Int. Power Electronics and Motion Control Conference (EPE/PEMC), 2012, pp. LS5b.2-1-LS5b.2-7.

[5] H. S. Che, W. P. Hew, N. A. Rahim, E. Levi, M. Jones, and M. J. Duran, "A six-phase wind energy induction generator system with seriesconnected DC-links," in 3rd IEEE Int. Symposium on Power Electronics for Distributed Generation Systems (PEDG), 2012, pp. 26-33.

[6] A. Tani, G. Serra, M. Mengoni, L. Zarri, G. Rini, and D. Casadei, "Dynamic stator current sharing in quadruple three-phase induction motor drives," in $39^{\text {th }}$ Annual Conference of the IEEE Industrial Electronics Society IECON, 2013, pp. 5173-5178.

[7] M. Mengoni et al., "Control of a fault-tolerant quadruple three-phase induction machine for more electric aircrafts," in 42nd Annual Conference of the IEEE Industrial Electronics Society IECON, 2016.

[8] R. Nelson and P. Krause, "Induction machine analysis for arbitrary displacement between multiple winding sets," IEEE Transactions on Power Apparatus and Systems, vol. PAS-93, no. 3, pp. 841-848, 1974.

[9] N. Trout, "A three-phase back-to-back alternator test," Students' Quarterly Journal, vol. 5, no. 20, pp. 194-197, 1935.

[10] D. Morris, "Back-to-back test for induction machines: motorised coupling," in Proceedings of the Institution of Electrical Engineers, 1967 , vol. 114, no. 8, pp. 1160-1161.

[11] W. Fong, "New temperature test for polyphase induction motors by phantom loading," in Proceedings of the Institution of Electrical Engineers, 1972, vol. 119, no. 7, pp. 883-887.

[12] M. Sheng and C. Grantham, "Synthetic loading of three-phase induction motors by magnetic field magnitude modulation," IEE Proceeding Electric Power Applications, vol. 141, no. 2, pp. 95-100, 1994.

[13] W. Soltani, B. Szabados, and G. Hoolboom, "A new synthetic loading for large induction machines with no feedback into the power system," IEEE Transactions on Energy Conversion, vol. 17, no. 3, pp. 319-324, 2002.

[14] A. Meyer and H. W. Lorenzen, "Two-frequency heat run - a method of examination for three-phase induction motors," IEEE Transactions on Power Apparatus and Systems, vol. PAS-98, no. 6, pp. 2338-2347, 1979.

[15] H. R. Schwenk, "Equivalent loading of induction machines for temperature tests," IEEE Transactions on Power Apparatus and Systems, vol. 96, no. 4, pp. 1126-1131, 1977.

[16] H. E. Jordan, J. H. Cook, and R. L. Smith, "Synthetic load testing of induction machines," IEEE Transactions on Power Apparatus and Systems, vol. 96, no. 4, pp. 1101-1104, 1977.

[17] S. L. Ho and W. N. Fu, "Analysis of indirect temperature-rise tests of induction machines using time-stepping finite element method," IEEE Power Engineering Review, vol. 21, no. 2, pp. 53-53, 2001.

[18] F. Luise, S. Pieri, M. Mezzarobba, and A. Tessarolo, "Regenerative testing of a concentrated-winding permanent-magnet synchronous machine for offshore wind generation --- Part I: Test concept and analysis," IEEE Transactions on Industry Applications, vol. 48, no. 6, pp. 1779-1790, 2012.

[19] F. Luise, S. Pieri, M. Mezzarobba, and A. Tessarolo, "Regenerative testing of a concentrated-winding permanent-magnet synchronous machine for offshore wind generation --- Part II: Test implementation and results," IEEE Transactions on Industry Applications, vol. 48, no. 6, pp. 1791-1796, 2012.

[20] I. Zoric, M. Jones, and E. Levi, "Arbitrary power sharing among threephase winding sets of multiphase machines," IEEE Transactions on Industrial Electronics, vol. 65, no. 2, pp. 1128-1139, Feb. 2018. 